\title{
Marked increase of final height by long-term aromatase inhibition in a boy with idiopathic short stature
}

\author{
Andreas Krebs ${ }^{1}$, Olaf Moske-Eick ${ }^{2}$, Jürgen Doerfer ${ }^{1}$, \\ Cordula Roemer-Pergher ${ }^{1}$, Natascha van der \\ Werf-Grohmann ${ }^{1}$ and Karl Otfried Schwab ${ }^{1, *}$ \\ ${ }^{1}$ Department of Pediatrics and Adolescence Medicine, \\ University Hospital, Freiburg, Germany \\ ${ }^{2}$ Department of Pediatric Radiology, University Children's \\ Hospital, Basel, Switzerland
}

\begin{abstract}
Growth hormone $(\mathrm{GH})$ is the most frequently used treatment in children with idiopathic short stature (ISS). Aromatase inhibitor (AI) therapy is still in an experimental state, and both final height $(\mathrm{FH})$ and long-term efficacy data in ISS have not been published. We present a 14.5-year-old boy with ISS and a height of $142.7 \mathrm{~cm}$ [standard deviation score (SDS) -2.79]. Based on the baseline bone age (BA) of 13.5-14 years, his predicted adult height (PAH) by Bayley/Pinneau was 154 cm (SDS -3.77)-158.2 (SDS -3.15). After a 5-year letrozole monotherapy, FH was $169 \mathrm{~cm}$ (SDS -1.57) showing a height difference between PAH and FH from 10.8 to $15 \mathrm{~cm}$. No permanent side effects of the medication have been observed. Both a transient occurrence and a spontaneous recovery of decreased bone mineral apparent density were seen, verified by dual-energy X-ray absorptiometry. Spinal magnetic resonance imaging revealed no vertebral abnormalities. AI therapy might be an effective and low-cost alternative to the use of GH. Further controlled trials should prove efficacy and safety of long-term AI therapy in boys with ISS.
\end{abstract}

Keywords: final adult height; idiopathic short stature; longterm aromatase inhibition; safety aspects.

\section{Introduction}

The diagnosis of idiopathic short stature (ISS) requires an individual height of at least -2 standard deviation score (SDS) below the population mean for age and sex, and

*Corresponding author: Prof. Dr. Karl Otfried Schwab, MD, Department of Pediatrics and Adolescence Medicine, University Hospital, Mathilden Street 1, D-79106 Freiburg, Germany Phone: +49-761-270 44820, Fax: +49-761-270 44140, E-mail: karl.otfried.schwab@uniklinik-freiburg.de Received October 19, 2011; accepted February 20, 2012; previously published online April 4, 2012 the absence of growth-related underlying diseases, such as endocrine disorders, malnutrition, chronic systemic diseases, or genetic defects (i.e., haploinsufficiency of the short stature homeobox-containing gene SHOX) (1). Concomitant counseling of children with ISS is recommended if they suffer from stigmatization, social isolation, chronic psychosocial stress, and their restricted quality of life (2). The importance of impairments related to short stature later in life is supported by a population-based study investigating the association between height and the risk of suicide in more than one million Swedish men. It could be shown that an increase in height of $5 \mathrm{~cm}$ was associated with a $9 \%$ decrease in suicide risk (3). Although psychosocial effects of short stature do not generally result in clinically significant problems of psychosocial adaptation, some patients suffer from it and consult frequently pediatric endocrinologists (2). Growth hormone $(\mathrm{GH})$ is the most frequently used medical treatment in children with ISS, which was approved in the United States by the Food and Drug Administration (FDA) in 2003 (1, 2), but the European Medicines Agency (EMEA) has not been given an approval for GH in ISS so far. The alternative aromatase inhibitor (AI) therapy, which has been shown to be effective in increasing predicted adult height (PAH) in children with ISS, is still at the experimental stage (4). However, the costs of treatment are considerably lower administering an AI than GH. The treatment with $\mathrm{GH}$ in children with ISS has resulted in a mean increase in adult height of $3.5-7.5 \mathrm{~cm}$ during a 4 - to 10 -year period of treatment (1). Previous treatment with the AI letrozole for 2 years has led to a PAH increase of $5.9 \mathrm{~cm}$ in boys with ISS (4). The main reasons for describing the case of a boy with ISS are a safe long-term letrozole monotherapy over 5 years resulting in a 10.8 - to $15-\mathrm{cm}$ height difference between PAH and final height $(\mathrm{FH})$ and the reversibility of decreased bone mineral apparent density (BMAD) 3 years after finishing the letrozole therapy.

\section{Patient report}

The 14.5-year-old boy was referred to pediatric endocrine evaluation because of severe short stature. There was no family history of endocrine or bone diseases. The mother was measured at $155 \mathrm{~cm}$ (SDS -1.87), and the father's height was $167 \mathrm{~cm}$ (SDS -2.01). The boy was born after 40 weeks of normal pregnancy and delivery to healthy, non-consanguineous parents with a birth weight of $3315 \mathrm{~g}$ (SDS -0.58) and a length of $49 \mathrm{~cm}$ (SDS -0.92). At initial presentation, the 
patient had a height of $142.7 \mathrm{~cm}(-2.79$ SDS) and a weight of $38.7 \mathrm{~kg}$, for a body mass index of $19 \mathrm{~kg} / \mathrm{m}^{2}$ (50th percentile for age and gender). His genetic target height by Tanner (5) was $167.5 \mathrm{~cm}$ (SDS -1.79 ), calculated by the equation: (father's height+mother's height) $/ 2 \pm 6.5 \mathrm{~cm}$. He was proportionate, had no dysmorphic features, and was pubertal, with Tanner stage 2 as to pubic hair and genital development. His testicular volume was $5 \mathrm{~mL}$. Estimated bone age (BA) was 13.5-14 years by Greulich/Pyle method. Therefore, PAH by Bayley/Pinneau method (6) was $154 \mathrm{~cm}$ (SDS -3.77) to 158.2 $\mathrm{cm}$ (SDS -3.15). Laboratory analyses including hepatic, renal, and endocrine function, sweat test, and celiac disease markers were normal.

\section{Results}

\section{Growth}

Treatment with the AI letrozole (Femara, Novartis AG, Switzerland) at a dose of $2.5 \mathrm{mg}$ daily was administered for 5 years. Based on the baseline BA of 13.5-14 years, the difference between PAH of 154 (SDS -3.77) to 158.2 (SDS -3.15) $\mathrm{cm}$ at the beginning and FH of $169 \mathrm{~cm}$ (SDS -1.57) at the end of the letrozole therapy was $10.8-15 \mathrm{~cm}$. FH was defined as height at an age $\geq 22$ years or height velocity $\leq 0.5 \mathrm{~cm}$ in the foregoing year. The height velocity during the 5-year follow-up period is shown in Figure 1. The patient did not report any

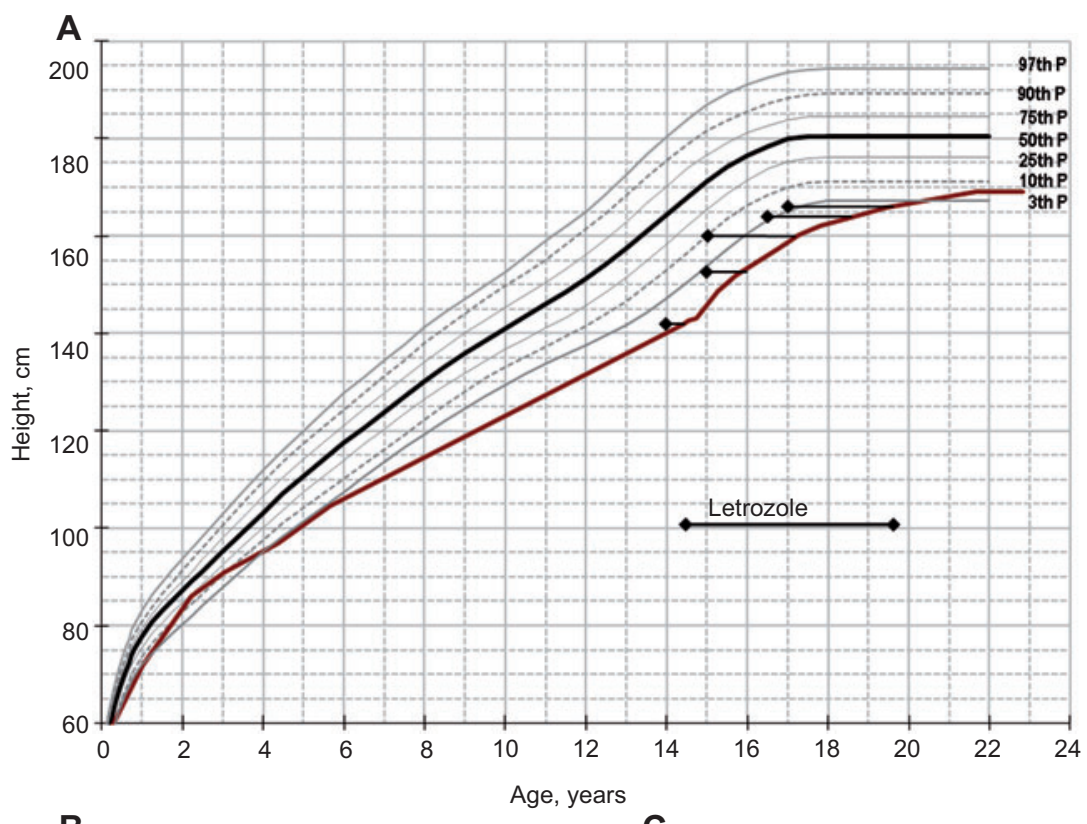

B
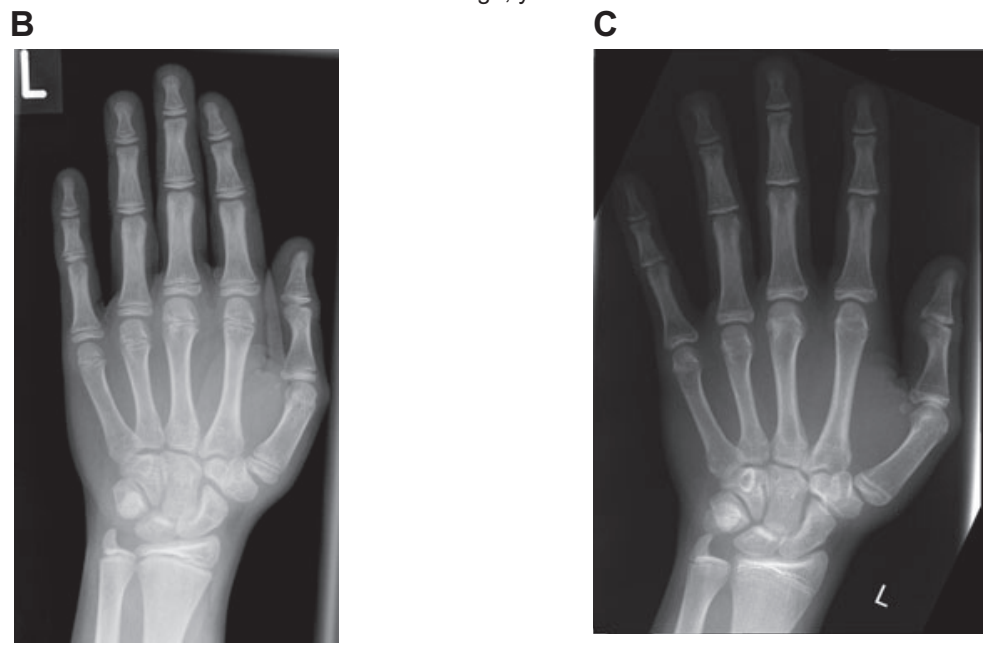

Figure 1 Growth chart (A) and X-ray pictures of the left hand (B, C) of a boy with ISS who was treated with letrozole (Femara, Novartis AG, Switzerland) at a dose of $2.5 \mathrm{mg}$ daily over a period of 5 years. Before treatment, the boy's height was $142.7 \mathrm{~cm}$ (SDS -2.79 ), his bone age according to Greulich/Pyle 13.5-14 years (Figure 1B), and his predicted adult height according to Bayley/Pinneau was 154 cm (SDS -3.77). Long-term letrozole treatment resulted in a final adult height of $169 \mathrm{~cm}$ (SDS -1.57). At the end of the letrozole treatment, the bone age according to Greulich/Pyle was 16 years (Figure 1C). The corresponding bone age values are shown as black rhombuses. P, percentile; L, left hand; SDS, standard deviation score. 
adverse effect. The progression of puberty was slightly accelerated with normal pubertal maturation. Tanner stage assessments of pubic hair and genital development were performed at least once a year. The boy had Tanner stage development 2 aged 14.5 (initiation of letrozole therapy), stage 4 aged 15.3, stage 5 aged between 15.9 and 19.5 (discontinuation of letrozole therapy). Testicular volume was $5 \mathrm{~mL}$ aged 14.5 (initiation of letrozole therapy), $15 \mathrm{~mL}$ aged 15.3, $20 \mathrm{~mL}$ aged 16.9, and $25 \mathrm{~mL}$ aged 19.5 (discontinuation of letrozole therapy).

\section{Laboratory determinations}

The patient was particularly monitored for endocrine, hepatic, and renal functions and showed no significant modification (Table 1).

\section{Radiological findings}

Repeated hand X-rays were performed and revealed an estimated BA by Greulich/Pyle method of 13.5-14 years at a chronological age of 14.5 years and a BA of 16 years at the end of the 5-year treatment period when the patient was 19.5 years old (see Figure 1). Hand bones showed no signs of demineralization or fractures. BA determinations were performed by two independent pediatric radiologists (O.M-E, M.U.).

In addition, spinal magnetic resonance imaging (MRI) of the patient was performed using a 1.5-Tesla Imager
(MR-Avanto, Siemens Medical Systems, Erlangen, Germany) in two steps. First, the imaging protocol of the cervical and upper thoracic spine. Second, the imaging protocol of the lower thoracic and lumbosacral spine. The composed sequences with 11 sagittal slices were performed with T1-weighted (field of view (FOV) $679 \times 379$ $\mathrm{mm}$, slice thickness $3 \mathrm{~mm}$, gap $10 \%=0.3 \mathrm{~mm}$, acquisition matrix $416 \times 746 \mathrm{~mm}$ ) and T2-weighted (FOV $681 \times 379$ $\mathrm{mm}$, slice thickness $3 \mathrm{~mm}$, gap $10 \%=0.3 \mathrm{~mm}$, acquisition matrix $486 \times 872 \mathrm{~mm}$ ) images. All protocols consisted of turbo-spin echo sequences for the assessment of vertebral anatomy, endplate abnormalities, and intervertebral disk. The MRI images were visually analyzed for vertebral anatomy and shape, endplate changes, disk height and water content, and muscle status of the back by a radiologist (OM-E). Measurements of vertebral body dimensions and assessments of the shape of each vertebral body were performed with a digital caliper in the radiologic workstation. Our patient showed no abnormalities in spinal MRI (Figure 2). The shapes of all vertebral bodies were classified as normal using the classification for pediatric vertebral body morphology (7).

\section{Bone mineral apparent density (BMAD)}

After discontinuation of letrozole treatment, examination of BMAD (8) with dual-energy X-ray absorptiometry (DXA) scanning (Lunar Prodigy Advance, GE Lunar Corp, Madison,

Table 1 Blood values prior, under, and after a 5-year treatment with the AI letrozole in a boy with idiopathic short stature.

\begin{tabular}{|c|c|c|c|c|}
\hline \multirow[t]{2}{*}{ Parameter } & \multicolumn{3}{|c|}{ Letrozole treatment } & \multirow[t]{2}{*}{ Reference values } \\
\hline & Prior & Under & After & \\
\hline Age, years & 14.5 & & 19.5 & \\
\hline Testosterone, $\mathrm{nmol} / \mathrm{L}^{\mathrm{a}}$ & 2.4 & 36.9 & 18.7 & $\begin{array}{l}12.2-41.6 \hat{\jmath} \text { Adults } \\
0.7-10.4 \hat{\jmath} \text { Adolescents }\end{array}$ \\
\hline $\mathrm{LH}, \mathrm{IU} / \mathrm{L}$ & 0.94 & 4.23 & 2.70 & $1.7-8.6$ \\
\hline FSH, IU/L & 1.0 & 3.0 & 1.30 & $1.5-12.4$ \\
\hline Estradiol, $\mathrm{pmol} / \mathrm{L}^{\mathrm{b}}$ & 58.7 & 139.5 & 136.1 & 27.9-191ठ̂ Adults \\
\hline Cortisol, nmol/L, a.m. & 226.2 & 146.2 & 446.9 & $171.0-535.2$ \\
\hline TSH, mIU/L & 1.12 & 1.22 & 1.84 & $0.27-4.20$ \\
\hline $\mathrm{AP}, \mathrm{U} / 1$ & 237 & 138 & 91 & $40-129$ \\
\hline IGF-I, nmol/L & - & - & 35.5 & $15.2-46.9$ \\
\hline Inhibin $B, n g / L$ & - & - & 363 & $120-400$ \\
\hline 25-OH-vitamin D, nmol/L & - & - & 58.5 & $50-175$ \\
\hline Creatinine, $\mu \mathrm{mol} / \mathrm{L}$ & 72.6 & 81.4 & 91.2 & $74.3-110.6$ \\
\hline$\gamma-\mathrm{GT}, \mathrm{U} / \mathrm{L}$ & 20 & 22 & 20 & $<60$ \\
\hline ALT, U/L & 18 & 25 & 18 & $<45$ \\
\hline Triglycerides, mmol/L & - & - & 1.02 & $0.29-2.28$ \\
\hline $\mathrm{TC}, \mathrm{mmol} / \mathrm{L}$ & - & - & 5.47 & $3.1-6.2$ \\
\hline LDL-C, mmol/L & - & - & 3.23 & $1.29-4.0$ \\
\hline $\mathrm{HDL}-\mathrm{C}, \mathrm{mmol} / \mathrm{L}$ & - & - & 1.37 & $1.03-2.06$ \\
\hline Hematocrit, \% & 41 & 44 & 46 & $33-61$ \\
\hline Hemoglobin, mmol/L & 9.0 & 10.0 & 10.1 & $8.1-9.9$ \\
\hline
\end{tabular}

${ }^{\mathrm{a} C h i r o n ~ D i a g n o s t i c s ~ A C S: ~ 180 T ~ t e s t o s t e r o n e ~ t e s t, ~ C h i r o n ~ D i a g n o s t i c s ~ G m b H, ~ D-35465 ~ F e r n w a l d, ~ G e r m a n y . ~}{ }^{\mathrm{b}}$ Chiron Diagnostics ACS: 180 Östradiol-6 II test. LH, luteinizing hormone; FSH, follicle-stimulating hormone; TSH, thyroid-stimulating hormone; AP, alkaline phosphatase; $\gamma$-GT, gamma glutamyltransferase; ALT, alanine aminotransferase; TC, total cholesterol; LDL-C, low-density lipoprotein cholesterol; HDL-C, high-density lipoprotein cholesterol. 

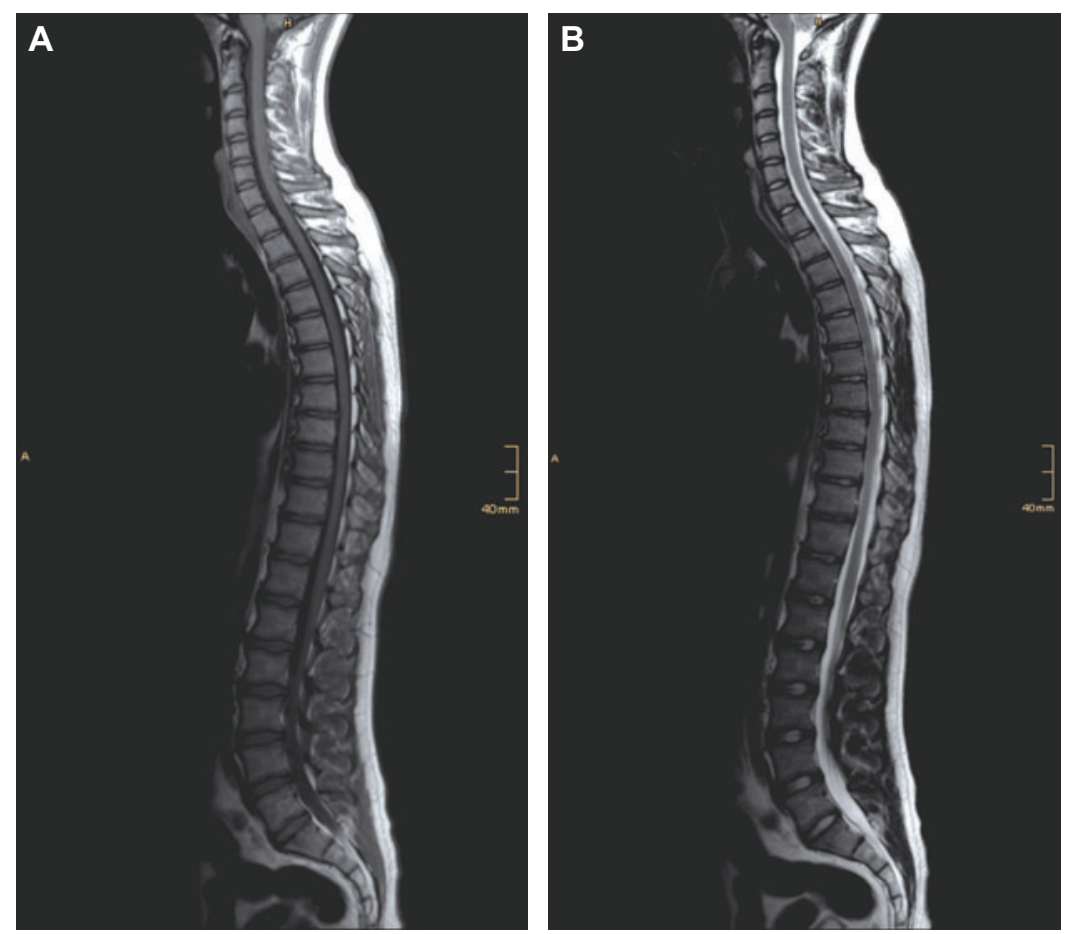

Figure 2 MR images of a 22.4-year-old man with ISS 3 years after the discontinuation of long-term letrozole treatment over 5 years. Sagittal T1-weighted MRI (A) of a composed spinal image and sagittal T2-weighted MRI (B) of a composed spinal image show normal vertebrae and intervertebral discs without any sign of vertebral abnormalities.

WI, USA) was carried out investigating the 2nd-4th vertebrae of the lumbar spine and the femoral neck. BMAD of the spine was $0.144 \mathrm{~g} / \mathrm{cm}^{3}$ (T-score: -0.84 ) and of the femoral neck 0.146 $\mathrm{g} / \mathrm{cm}^{3}$ (T-score: -0.89 ). After a 3-year ambulatory care without any medication, the bone mineral content values returned to normal, and BMAD of the spine was $0.168 \mathrm{~g} / \mathrm{cm}^{3}$ (T-score: 0.37 ) and of the femoral neck $0.174 \mathrm{~g} / \mathrm{cm}^{3}$ (T-score: 0.11 ).

\section{Discussion}

Based on the BA of 13.5-14 years, the present casuistics describes a height difference from 10.8 to $15 \mathrm{~cm}$ between PAH and an FH after a 5-year AI monotherapy with letrozole in a pubertal boy with ISS. To date, AI therapy is still at the experimental stage (4), and no long-term efficacy and adult height data in males with ISS have been published. An improvement in PAH by $5.9 \mathrm{~cm}$ during a 2-year treatment with letrozole (2.5 mg, once daily) in 31 boys with ISS, aged 9.0-14.5 years, could be shown in a placebo-controlled Finnish study (9). Therefore, our case report might be a helpful hint indicating the possibility of increasing FH in males with ISS by longterm AI therapy.

In comparison with $\mathrm{GH}$ monotherapy up to 10 years, AI monotherapy might not only be equally effective in increasing PAH and adult height in boys with ISS but also a lowcost alternative $(1,4,9)$. Future well-controlled studies in children with ISS should involve long-term AI treatment with regard to adult height outcome and safety. In terms of AI therapy, some potential safety concerns exist. Highdensity lipoprotein cholesterol could decrease, but sufficient data are still lacking regarding the influence of aromatase inhibition on spermatogenesis, cognitive function, vascular wall function, and prostate growth (4). Short-term AI therapy in ISS has proved to be well tolerated, but the number of patients who are treated within clinical trials is small (1, $4,9)$. In patients who may receive long-term AI therapy, bone health is of particular interest. AI treatment prevents biosynthesis of estrogen, thereby slowing its efficacy on skeletal maturation, especially epiphyseal closure and bone mineralization $(1,4)$. Transiently reduced BMAD after a 5 -year letrozole therapy in our adolescent boy may support the importance of monitoring bone health during AI therapy. But the spontaneous recovery also shows that no permanent harm is to be expected. Hero et al. reported mild vertebral body deformities in the thoracic spine (MRI) in 5 of 11 boys with ISS (mean age 10.7 years) who received letrozole for 2 years during prepuberty or early puberty (10). The authors discussed the possibility that the use of AI treatment during prepuberty may be associated with an increased risk of vertebral deformities. Although our patient was during midpuberty and 4 years older than the boys with ISS described by Hero et al., we carefully assessed his vertebral morphology by spine MR imaging, but no abnormalities could be found. In adolescent males with GH deficiency, an increase in adult height potential was seen by adding an AI to GH therapy. Normal pubertal progression and bone health were found after 2-3 years with this combination therapy (11). Another 
randomized, placebo-controlled study could show that boys with constitutional delay of puberty who received the AI letrozole in combination with low-dose testosterone reached a higher mean near-final height than boys on testosterone monotherapy (12). Considering the positive effects of $\mathrm{GH}$ on BMD, the use of $\mathrm{GH}$, together with an AI, might also be a treatment option in adolescents with ISS.

In conclusion, this case involves a 14.5-year-old boy with ISS who achieved a height increase between PAH and FH from 10.8 to $15 \mathrm{~cm}$ during a 5-year AI therapy. In addition, the boy showed both a transient occurrence and a spontaneous recovery of decreased BMAD after long-term AI therapy, verified by DXA. MRI revealed no vertebral morphology abnormalities. Any other measures regarding health and safety monitoring showed no pathology. Therefore, AI therapy might be an effective and low-cost alternative to the use of GH. However, these findings deserve further attention, and controlled long-term studies are necessary to prove safety and efficacy of AI treatment in males with ISS.

\section{References}

1. Cohen P, Rogol AD, Deal CL, Saenger P, Reiter EO, et al., on behalf of the 2007 ISS Consensus Workshop participants. Consensus statement on the diagnosis and treatment of children with idiopathic short stature: a summary of the Growth Hormone Research Society, the Lawson Wilkins Pediatric Endocrine Society, and the European Society for Paediatric Endocrinology Workshop. J Clin Endocrinol Metab 2008;93:4210-7.

2. Bullinger M, Koltowska-Häggström M, Sandberg D, Chaplin J, Wollmann $\mathrm{H}$, et al. Health-related quality of life of children and adolescents with growth hormone deficiency or idiopathic short stature - part 2: available results and future directions. Horm Res 2009;72:74-81.
3. Magnusson PK, Gunnell D, Tynelius P, Smith GD, Rasmussen F. Strong inverse association between height and suicide in a large cohort of Swedish men: evidence of early life origin of suicidal behavior? Am J Psychiatry 2005;162:1373-5.

4. Dunkel L. Update on the role of aromatase inhibitors in growth disorders. Horm Res 2009;71:57-63.

5. Tanner JM, Goldstein H, Whitehouse RH. Standards for children's height at ages 2-9 years allowing for height of parents. Arch Dis Child 1970;45:755-62.

6. Bayley N, Pinneau SR. Tables for predicting adult height from skeletal age: revised for use with the Greulich-Pyle hand standards. J Pediatr 1952;40:423-41.

7. Mäkitie O, Doria AS, Henriques F, Cole WG, Compeyrot S, et al. Radiographic vertebral morphology: a diagnostic tool in pediatric osteoporosis. J Pediatr 2005;146:395-401.

8. Katzman DK, Bachrach LK, Carter DR, Marcus R. Clinical and anthropometric correlates of bone mineral acquisition in healthy adolescent girls. J Clin Endocrinol Metab 1991;73: 1332-9.

9. Hero M, Norjavaara E, Dunkel L. Inhibition of estrogen biosynthesis with a potent aromatase inhibitor increases predicted adult height in boys with idiopathic short stature: a randomized controlled trial. J Clin Endocrinol Metab 2005;90: 6396-402.

10. Hero M, Toiviainen-Salo S, Wickman S, Mäkitie O, Dunkel L. Vertebral morphology in aromatase inhibitor-treated males with idiopathic short stature or constitutional delay of puberty. J Bone Miner Res 2010;25:1536-43.

11. Mauras N, Gonzalez de Pijem L, Hsiang HY, Desrosiers P, Papaport R, et al. Anastrozole increases predicted adult height of short adolescent males treated with growth hormone: a randomized, placebo-controlled, multicenter trial for one to three years. J Clin Endocrinol Metab 2008;93:823-31.

12. Hero M, Wickman S, Dunkel L. Treatment with the aromatase inhibitor letrozole during adolescence increases near-final height in boys with constitutional delay of puberty. Clin Endocrinol (Oxf) 2006;64:510-3. 